Original paper

\title{
Dosimetric characterization of a small-scale (Zn,Cd)S:Ag inorganic scintillating detector to be used in radiotherapy
}

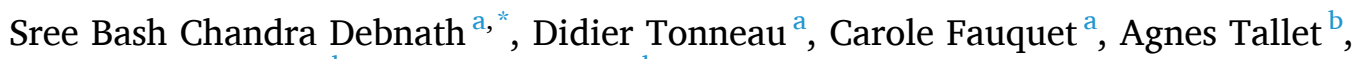 \\ Anthony Goncalves ${ }^{\mathrm{b}, \mathrm{c}}$, Julien Darreon ${ }^{\mathrm{b}}$ \\ ${ }^{\text {a }}$ Aix Marseille Université, CNRS, CINAM UMR 7325, 13288 Marseille, France \\ ${ }^{\mathrm{b}}$ Institut Paoli-Calmettes, 13009 Marseille, France \\ ${ }^{\mathrm{c}}$ Aix Marseille Université, CNRS UMR 7258, INSERM UMR 1068, CRCM, 13009 Marseille, France
}

\section{A R T I C L E I N F O}

\section{Keywords:}

Inorganic scintillating detector

Dosimetry

Small field dosimetry

Radiotherapy

\begin{abstract}
A B S T R A C T
Purpose: In modern radiotherapy techniques, to ensure an accurate beam modeling process, dosimeters with high accuracy and spatial resolution are required. Therefore, this work aims to propose a simple, robust, and a smallscale fiber-integrated X-ray inorganic detector and investigate the dosimetric characteristics used in radiotherapy.

Methods: The detector is based on red-emitting silver-activated zinc-cadmium sulfide (Zn,Cd)S:Ag nanoclusters and the proposed system has been tested under $6 \mathrm{MV}$ photons with standard dose rate used in the patient treatment protocol. The article presents the performances of the detector in terms of dose linearity, repeatability, reproducibility, percentage depth dose distribution, and field output factor. A comparative study is shown using a microdiamond dosimeter and considering data from recent literature.

Results: We accurately measured a small field beam profile of $0.5 \times 0.5 \mathrm{~cm}^{2}$ at a spatial resolution of $100 \mu \mathrm{m}$ using a LINAC system. The dose linearity at $400 \mathrm{MU} / \mathrm{min}$ has shown less than $0.53 \%$ and $1.10 \%$ deviations from perfect linearity for the regular and smallest field. Percentage depth dose measurement agrees with microdiamond measurements within $1.30 \%$ and $2.94 \%$, respectively for regular to small field beams. Besides, the stem effect analysis shows a negligible contribution in the measurements for fields smaller than $3 x 3 \mathrm{~cm}^{2}$. This study highlights the drastic decrease of the convolution effect using a point-like detector, especially in small dimension beam characterization. Field output factor has shown a good agreement while comparing it with the microdiamond dosimeter.

Conclusion: All the results presented here anticipated that the developed detector can accurately measure delivered dose to the region of interest, claim accurate depth dose distribution hence it can be a suitable candidate for beam characterization and quality assurance of LINAC system.
\end{abstract}

\section{Introduction}

In clinical medicine, high energy X-rays are used in radiotherapy to treat cancer, to detect and damage malignant tumors [1], where dosimetry plays an important role in modern radiation oncology [2]. The dose measurements at high energy are conventionally carried out by thermoluminescent dosimeters (TLD), ionization chambers, semiconductor devices (silicon diodes, MOSFETs, etc.), Gafchromic films, or with optically stimulated plastic dosimeters [3-8].TLD techniques require a long readout procedure, leading to a time-consuming process. Ionization chambers are most accurate for large fields but are challenging for field sizes below $2 \times 2 \mathrm{~cm}^{2}$, because of their large sensitive volume [9], anomalous polarity effect/polarity correction [10,11], and orientation-dependent responses that arise several corrections factors and require further investigations [11,12]. Owing to their small size, MOSFETs are generally used for in-vivo dosimetry but they have a short lifespan, exhibit energy and directional dependence with a poor signalto-noise ratio (SNR), and reproducibility [13]. Diode sensors are known to over-respond to low energy photons, show variations of sensitivity with the accumulated absorbed dose [14]. Unshielded diodes ('electron diodes') potentially offer good spatial resolution dosimetry but require significant corrections for field sizes below $1 \mathrm{~cm}[15,16]$. In addition,

\footnotetext{
* Corresponding author.

E-mail address: sree.debnath@univ-amu.fr (S.B.C. Debnath).
} 
shielded diodes ('photon diodes') contain high-density material (e.g., tungsten), and their response is not completely independent of field size [14].

Moreover, most of these detectors are not advisable for small field dosimetry due to the necessary corrections of volume averaging effects, lack of charge particle equilibrium, and dose perturbation [5,12,17]. Though some research studies have shown that radiographic/radiochromic films are suitable for small field dosimetry [18,19], they still require a significant time-consuming processing phase and have orientation dependency $[12,20]$. Synthetic diamond detectors are considered as suitable detectors due to their small sensitive volume, directional independence [21], high dose-response [22], and they are suitable for accurate reference dosimetry down to $0.5 \times 0.5 \mathrm{~cm}^{2}$ field size [23]. However, they require correction factors due to absorbed dose rate dependency [12], orientation type [21], and comparable low lateral resolution due to significant head size while using face-on orientation. Therefore, a ubiquitous demand in recent medical dosimetry is to develop a reliable, high-resolution, and sensitive detector to be useful in radiotherapy to characterize small fields and fields with high gradients. Consequently, some commercial detectors (e.g., exradin W1 and W2) based on scintillating materials emerged since the beginning of 2010 $[24,25]$.

The idea of using optical fiber for radiation dose monitoring has been studied by different research groups [26-30]. Different research works in this direction have shown that the luminescent signal emitted by the scintillators is proportional to the absorbed dose, and the signal is almost independent of photon energy in the megavolt (MV) range $[2,7,31,32]$. Scintillators used in these dosimeter techniques can be used for absorbed dose determination under high energy irradiation. These detectors can be manufactured in small dimensions that could provide linear response to dose, dose rate proportionality, energy independence, and the benefit of having near water equivalent plastic scintillators $[31,33]$. However, the main difficulties in the use of scintillators are low signal-to-noise (SNR), degradation of signal with accumulated dose, a minimum size required, and the correction factors necessary due to the significant stem light generation in wide core fiber cable $[12,33]$.

In this context, we developed a small-scale X-ray inorganic scintillating detector (ISD) based on (Zn,Cd)S:Ag scintillating clusters to characterize high energy radiation beam with few centimeters to few millimeters field dimension. The feasibility of the detector was verified through real-time measurement of several dosimetric parameters. A comparison with a commercial dosimeter (PTW microdiamond) is presented. Such a detector is commonly used for regular to small field radiotherapy treatment in the service.

\section{Materials and methods}

\section{Sensor design and principle of measurement}

The developed novel X-ray detector essentially consists of a silica $\left(\mathrm{SiO}_{2}\right)$ optical fiber (ref. FG050UGA) equipped with scintillator clusters grafted at the fiber extremity. The fiber end was sharply cleaved by a cleavage system before attaching the scintillator. Fiber core and cladding diameters used are respectively $50 \mu \mathrm{m}$ and $125 \mu \mathrm{m}$ (Thorlabs ${ }^{\mathrm{TM}}$ ), respectively. Scintillating clusters, made of (Zn,Cd)S:Ag powder (ref. JGL47/S-R1- $6 \mu \mathrm{m}$ median particle size) supplied by Phosphor Technology $\odot$ are embedded at the fiber extremity, which follows the same techniques used in our previous work using silver-doped ZnS scintillator [34]. (Zn,Cd)S:Ag scintillator was chosen due to its efficient and stable red emission tested [30]. The maximum sensitive surface of the ISD cross-section is limited by fiber cladding surface $\left(0.016 \mathrm{~mm}^{2}\right)$ rather than by core $\left(0.01 \mathrm{~mm}^{2}\right)$. Indeed, under hard X-ray excitation, the luminescence generated by one outer grain facing the narrow cladding part cannot be collected by the fiber core. However, the same grain reemits lower energy $\mathrm{x}$-rays that can excite grains facing the fiber core. Moreover, the visible light emitted by grains located far from the fiber core input is not collected by the core because of both reabsorption (weak phenomenon) and diffusion by surrounding grains (most probable phenomenon). Thus, the efficient detector head can be considered as a cylinder prolonging the fiber cladding, though the efficient volume is more complex to quantify. Under the exposure to high energy X-ray, the scintillator emits visible luminescence at around $550 \mathrm{~nm}[35,36]$ that penetrates the fiber core and propagates through the fiber. The other extremity of the fiber is plugged to a photon counter (ref. SPD_A_VIS_M1- Aurea Technology ${ }^{\mathrm{TM}}$ ) by means of a FC/PC connector (ref. B30230C), collecting the visible photons transmitted through the fiber. The photon counter is remotely controlled and measures the visible luminescence flux in photons per second. Note that, to ensure a maximum light signal transfer from the scintillator to the counter, a sharp cleaving system is applied. The detector is in-lab tested at each step of the fabrication process to ensure the cleavage and cluster grafting qualities with eminent quantum yield.

Due to the highly sensitive photon counter ( 20 ns gate width at $14 \mathrm{ps}$ sampling time), the ISD conveys low rise-time and fast responses to the irradiation. Fig. 1 represents the active part of the ISD.

In the experimental environment, ambient light coming from surroundings leads to an average background noise varying from 150 photons/s to 450 photons/s depending on environmental conditions. Thus, the exact value of the average ambient noise was measured during experiments and systematically removed from each measurement.

\section{Experimental setup}

The overall proposed radiation measurement system is shown in Fig. 2. A testbed was developed on the patient support system available at radiotherapy service under an Elekta Synergy LINAC system capable of delivering both $6 \mathrm{MV}$ and $15 \mathrm{MV}$ photons. The LINAC multi-leaf collimator (MLC) and lateral jaws system can be set up to vary the irradiation field from $30 \times 30 \mathrm{~cm}^{2}$ down to $0.5 \times 0.5 \mathrm{~cm}^{2}$. For a specific field characterization, the collimator set-up was not reset during the

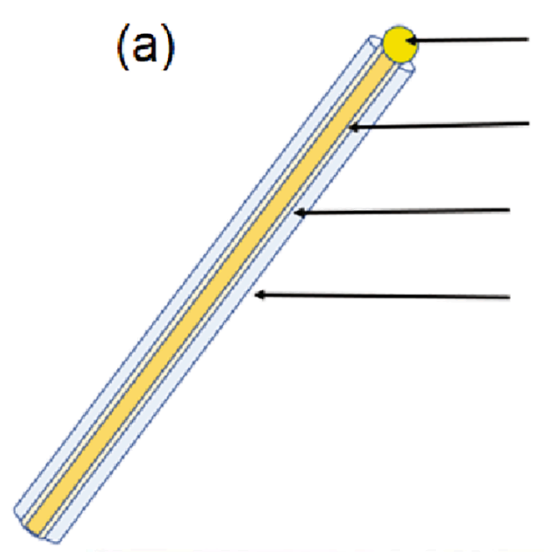

Scintillating clusters ball

Glass optical fiber $(50 \mu \mathrm{m}$ core diameter)

Fiber cladding $(125 \mu \mathrm{m}$ coating diameter)

Fiber outer Coating ( 250 $\mu \mathrm{m}$ diameter)

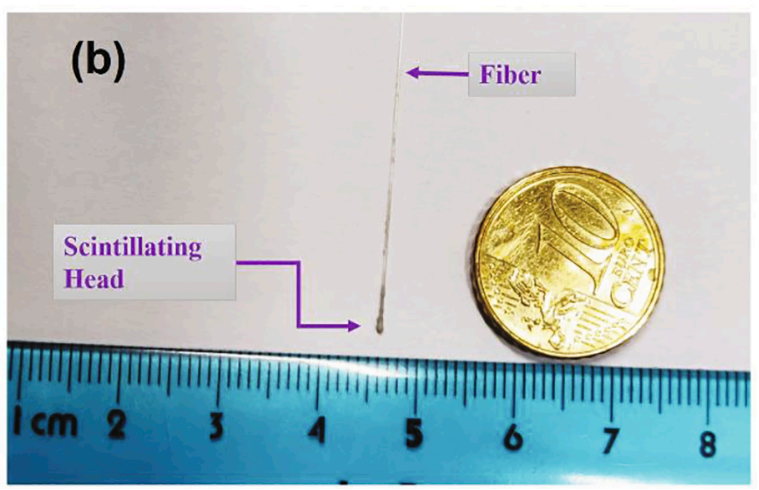

Fig. 1. (a) Schematic representation of the detector and (b) actual detector (active end). 
(Radiotherapy room)

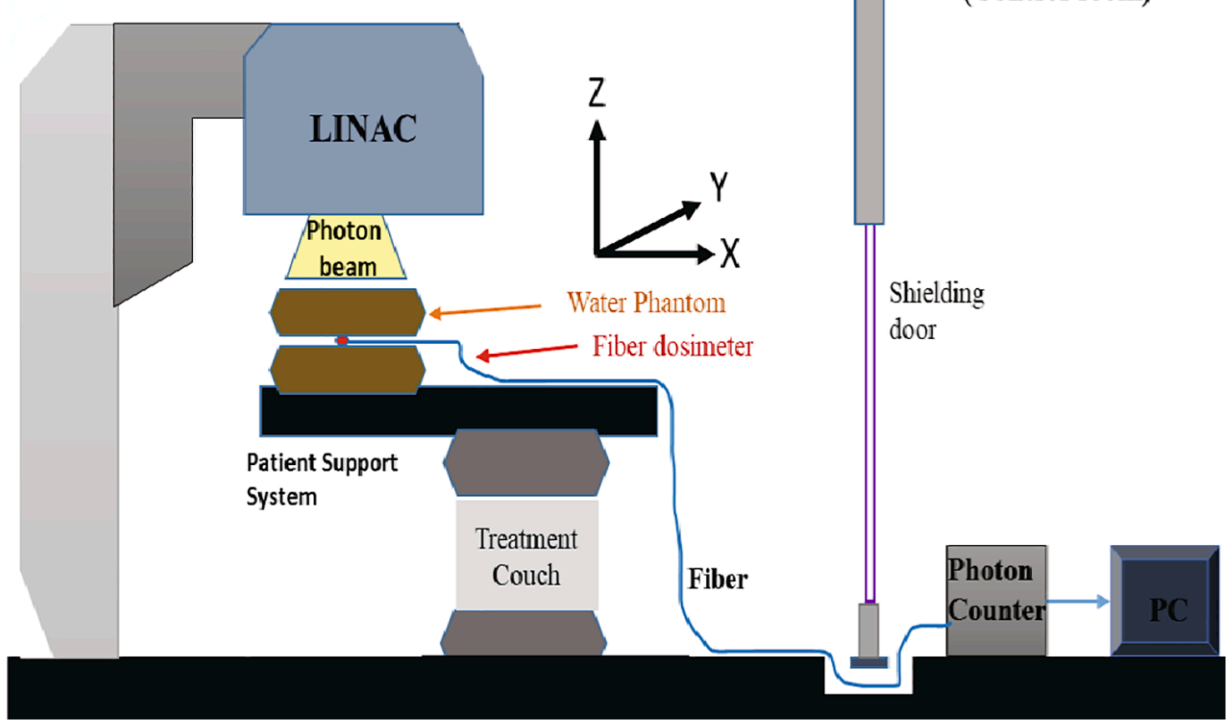

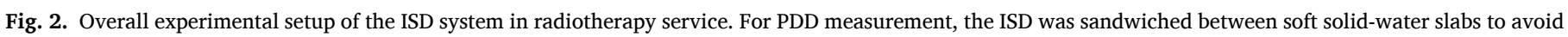
any possible air-gaps between slabs. Later, solid phantoms were replaced by water tank (IBA ${ }^{\circledR}$ ) phantom for all other measurements.

measurement. The photon counter is positioned at about $8 \mathrm{~m}$ from the detector sensitive head to avoid any interactions with X-ray. The whole setup is remotely controlled from an external control room avoiding any exposure of the electronics to high-energy irradiation.

A standard setup on the patient support assembly comprising a motorized X-Y-Z piezo stage allows characterizing small field beams at high spatial resolution. Consequently, the sensitive head of the detector is fixed to the motorized stack and the high-resolution 3D piezo controller ( $\sim 20 \mathrm{~nm}$ step) allows moving the detector head across the field with a selective step size. In order to compare the ISD performances with the reference microdiamond dosimeter, the preceding $\mathrm{X}-\mathrm{Y}-\mathrm{Z}$ piezo stage was replaced by a water tank (IBA®) equipped with a $3 D$ translation stages of $100 \mu \mathrm{m}$ minimum step size. During the experiment, solid water slabs of $30 \times 30 \mathrm{~cm}^{2}$ size were also used to confirm the depth dose distribution measurement in the water tank.

\section{Stem effect and background analysis}

The major drawbacks of conventional optical fiber-based scintillating detectors are their high sensitivity to 'stem signal', considered as a noise introducing an offset in the dose measurement. This effect includes both Cerenkov and direct fiber fluorescence contribution. When hardcore silica optical fiber is irradiated with high energy X-ray $(>125$ $\mathrm{kV}$ ), the dominant part of the stem signal is coming from the Cerenkov light generation. This latter effect is observed when generated high energy charged particles (e.g., electrons) penetrate a medium at speed faster than light and leading to extra light emission. Therefore, the stem signal must be characterized while a reliable estimation of the dose is required, and several techniques have been proposed to diminish this contribution from signal amplitude [27,37].

In this study, a background fiber method $[33,37,38]$ was considered as the simplest way to quantify and remove the contribution of this effect. This method consists of having a blank fiber without scintillators in parallel with the actual detector to estimate the Cerenkov light generation. The ISD and blank fiber extremities are maintained at the field center and placing the optical fiber axis perpendicularly to the beam axis. It relies on the assumption that the Cerenkov signal in the background fiber is the equal magnitude of the signal fiber. Finally, the actual signal of the scintillator that is equivalent to the irradiated dose is obtained by subtracting the signal of the background fiber from the total signal provided by the scintillating detector.

\section{Dosimetric characteristics}

The ISD has been used to demonstrate several dosimetry properties, and a comparison was shown with the microdiamond dosimeter. Unless otherwise stated, all the measurements have been performed online using standard source-to-surface distance (SSD) of $90 \mathrm{~cm}$ and source-toaxis distance $(\mathrm{SAD})$ of $100 \mathrm{~cm}$ at the central axis beam isocenter. The measurement uncertainties were reported based on A-type standard uncertainty estimation (statistical analysis) [39]. The total uncertainties reported for the experimental results include repeatability of the measurement and detector's positioning uncertainty (grouped by type B).

\section{Beam profiling}

The ISD system was used to perform high spatial resolution beam profiling for the smallest field available at the LINAC $\left(0.5 \times 0.5 \mathrm{~cm}^{2}\right)$ both in inline and crossline directions. First, beam profiling was made in the air with a high-resolution 3D stepper motor. This also allows characterizing convolution effect, which usually appears during small field beam profiling. Later, to show the comparison with the microdiamond dosimeter, beam profiling was made inside water phantoms under 3D translation stages attached to the blue water phantom (IBA ${ }^{\mathrm{TM}}$ ). Note that, collimators were not reset during measurements, and measurements were made three times at each phase. Thus, all the experiments were performed using the LINAC system used for clinical treatment. Because the aim here is to compare the performances of two different detectors in terms of convolution effect contribution in the raw measurements during small field characterizations, we chose to present field profiling along only one axis (e.g., crossline).

\section{Stability}

The ISD system stability was tested employing repeatable measurement at the same position for several consecutive and day-to-day measurements. Certainly, measurement stability also integrates the stability of the collimators and the LINAC itself. In order to assess the short-term repeatability, the measurement was tested under regular $\left(10 \times 10 \mathrm{~cm}^{2}\right)$ and small field $\left(0.5 \times 0.5 \mathrm{~cm}^{2}\right)$ size at a constant high dose rate of 400 $\mathrm{MU} / \mathrm{min}$ over 1-hour irradiation. The long-term daily reproducibility of the detector was tested as well by measuring the scintillating signal on eight consecutive days in the same environment and the identical location of the detector for $10 \times 10 \mathrm{~cm}^{2}$, and $0.5 \times 0.5 \mathrm{~cm}^{2}$ beam fields. During these measurements, the ISD was plugged and unplugged every 
day as well as the entire set-up mounted and unmounted. Each time the measurement was made for $1 \mathrm{~Gy}$ dose delivered with the photon energy under the 6MV LINAC beam. The error of each measurement point was calculated by the following equation of standard deviation:

$\sigma_{s}=\sqrt{\frac{1}{n} \sum\left(x_{i}-\bar{x}\right)^{2}}$

Where $x_{i}$ represents the signal intensity measured at time $t_{i}, \bar{x}$ is the average value of the ripple, and $\mathrm{n}$ is the number of measurement points.

\section{Dose linearity}

Detector's linear response to the dose was tested as a function of radiation dose ranging from $4 \mathrm{MU}$ ( $4 \mathrm{cGy}$ ) up to $500 \mathrm{MU}$ ( $500 \mathrm{cGy}$ ) at a constant dose rate of $400 \mathrm{MU} / \mathrm{min}$. The upper bound was chosen as the maximum stable dose rate of the machine tested during irradiation. For each measurement, the dose was delivered at $6 \mathrm{MV}$ within field sizes of $10 \times 10 \mathrm{~cm}^{2}$ and $1 \times 1 \mathrm{~cm}^{2}$ by placing the detector under standard reference condition defined by TRS 398 [40]. To fully test the linearity, the data were normalized to 100 cGy dose (linearity index 1 to $100 \mathrm{cGy}$ ), and then a linear fit was used to see how much values deviate from the linearity index.

\section{Percentage depth dose}

In clinical practice, the percentage depth dose (PDD) allows estimating the central axis dose distribution in the region of interest inside a human body. Hence, PDD was performed for various field sizes of $10 \times$ $10 \mathrm{~cm}^{2}, 5 \times 5 \mathrm{~cm}^{2}, 3 \times 3 \mathrm{~cm}^{2}, 2 \times 2 \mathrm{~cm}^{2}, 1 \times 1 \mathrm{~cm}^{2}$, and $0.5 \times 0.5 \mathrm{~cm}^{2}$. These measurements were carried out from the surface down to a 200 $\mathrm{mm}$ water equivalent depth keeping SSD at $100 \mathrm{~cm}$. To confirm the measurement accuracy, data measurements were made both in solid water phantoms and water tank phantoms. A comparison of PDD measurements was shown and discussed.

\section{Relative dose response}

The radiation dose response by the ISD was measured by correlating the measured optical signal (photons) with the respective delivered dose in the above-mentioned reference conditions. A calibration for the ISD was made at LINAC isocenter with $10 \times 10 \mathrm{~cm}^{2}$ field size and $10 \mathrm{~cm}$ water depth, which suggests that for 100 cGy dose, the ISD measures $8.4 \times 10^{5}$ total integrated photons at the photon counter. The relative dose for the ISD was measured based on this calibration coefficient. All the measurements have been carried out at a fixed $100 \mathrm{MU}$ dose (1 MU 1 cGy in TRS 398 reference conditions) delivered at a typical dose rate of 400
$\mathrm{MU} / \mathrm{min}$ and $6 \mathrm{MV}$ photons.

\section{Field output factor}

Field output factor (OF) is one of the major concerns in small field radiotherapy treatment and exhibits some complexities when measurements are carried out with the existing conventional dosimeters. This output factor should be considered to realize the signal dependency on different field sizes [12,25], to avoid potential errors that sometimes lead to serious consequences for patient care. The optical signal for different beam fields was measured at 100 cGy dose maintaining the sensor head at the standard reference condition described before. Field output factors for microdiamond dosimeter and the ISD were measured according to the reference condition defined by TRS-483 and they were presented in terms of effective (measured) field size [41]. The measurements were repeated three times, and both in-plane and cross-plane profiles were considered to calculate effective field size.

\section{Results}

\section{High resolution beam profiling and comparison}

Fig. 3 (a) presents the field profile recorded with SAD of $100 \mathrm{~cm}$ in the air by displacing the detector head across the lateral field within the crossline plane with $100 \mu \mathrm{m}$ step size. The profile shows a gaussian-like variation with a Full Width at Half Maximum (FWHM) of about $5.3 \mathrm{~mm}$. Fig. 3 (b) represents the crossline profile of the same selected field inside the water tank phantoms (IBA®) at $10 \mathrm{~cm}$ water depth with SSD $90 \mathrm{~cm}$. It shows the ISD performance in comparison with the microdiamond detector. Because the physical principle of these detectors is different, curves have been normalized at the maximum value. Both profiles represent an identical Gaussian shape with FWHM of $6 \mathrm{~mm}$ and $7 \mathrm{~mm}$ respectively for the ISD and microdiamond. The measurement is in good agreement everywhere except in the penumbra region (maximum percentage difference $\sim 17 \%$ ), which is mainly due to the volume averaging effect of the microdiamond owing to its larger sensitive head. The average percentage difference was found to be $\sim 1.5 \%$, which is still better than our previous results [34]. Finally, far from the field center ( $\geq 6 \mathrm{~mm}$ ), both detectors present almost the same normalized response.

\section{Repeatability and Reproducibility}

Fig. 4 shows the normalized value of total visible photons detected during ten successive measurements of $100 \mathrm{cGy}$ dose irradiations. In this

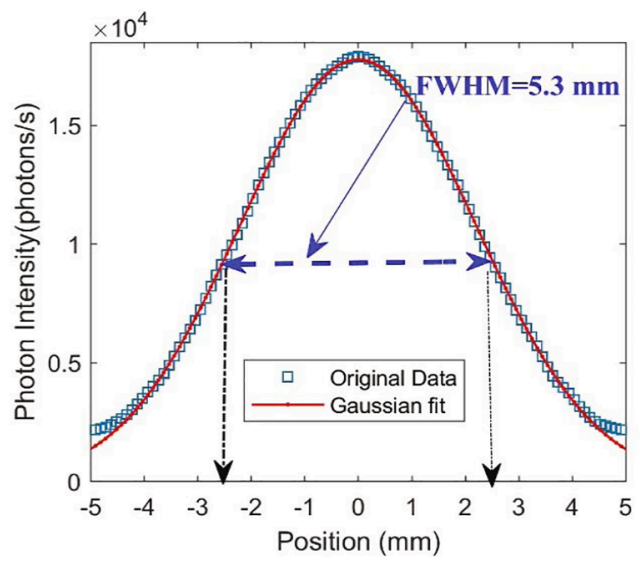

(a)

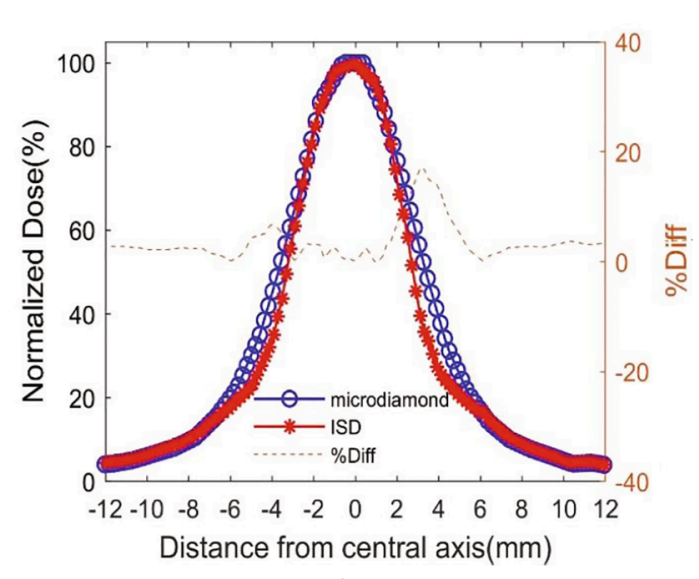

(b)

Fig. 3. (a) Small field $\left(0.5 \times 0.5 \mathrm{~cm}^{2}\right)$ beam profile measured in air within the crossline plane by the ISD. (b) Beam profile of $0.5 \times 0.5 \mathrm{~cm}^{2}$ field obtained using the ISD and microdiamond. In brown is shown the percentage difference between the two data sets. Brown (dotted line) curve refers to the right-hand side scale, whereas the blue and red curve refers to the left-hand side scale. (For interpretation of the references to color in this figure legend, the reader is referred to the web version of this article.) 

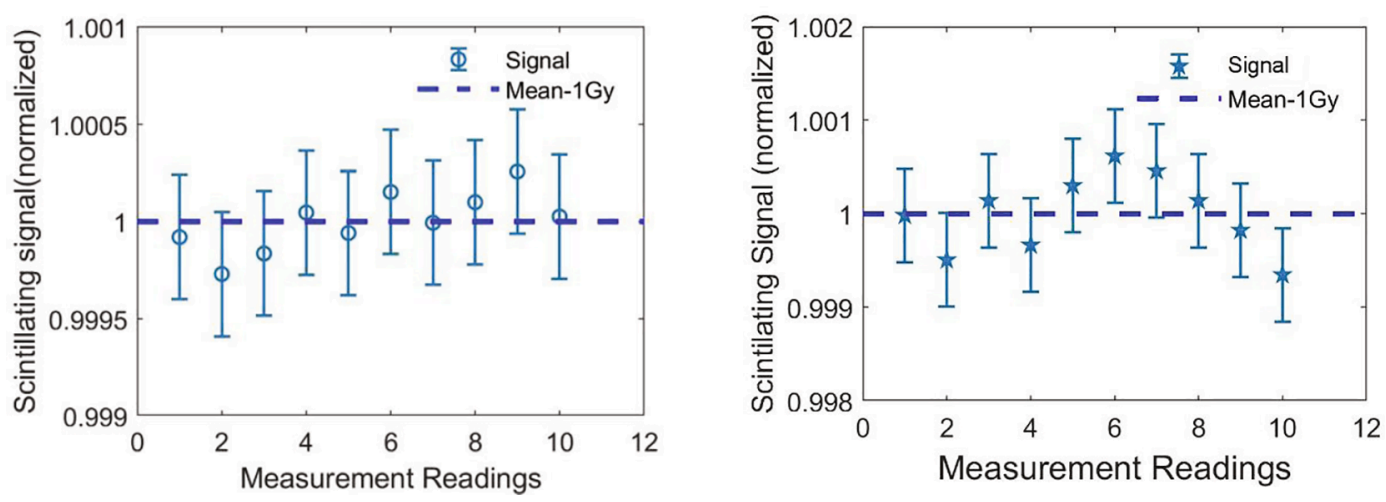

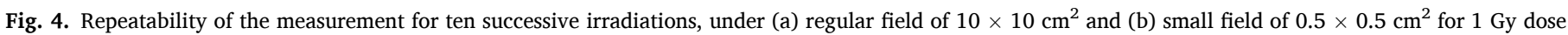
delivered at $6 \mathrm{MV}$. Measurements normalized to an average of 1 .

case, each measurement was performed repeatedly giving 5 min pause in-between, and the associated signal was recorded at the photon counter after each irradiation. These results illustrate that the stability of the ISD while measuring total scintillating signal as equivalent dose and resulted in a standard error of 0.0001 for $10 \times 10 \mathrm{~cm}^{2}$ field and 0.00015 for $0.5 \times 0.5 \mathrm{~cm}^{2}$ field, respectively. The ISD system demonstrates excellent repeatability with the maximum deviation of $0.02 \%$, and $0.07 \%$ from its average value calculated at $1 \mathrm{~Gy}$ dose for $10 \times 10 \mathrm{~cm}^{2}$, and $0.5 \times 0.5 \mathrm{~cm}^{2}$ beam fields, respectively. Error bars were estimated from the signal standard deviation following equation (1).

After testing the day-to-day reproducibility as explained in section 2.4.2, the system did not vary more than $0.1 \%$ from the mean value of daily irradiation while repeated over eight consecutive days as shown in Fig. 5. The standard error of the total scintillating signal is determined to be 0.00021 . This represents a good reproducibility of the ISD and stability of the system.

\section{Dose linearity}

During the dose linearity test, the integrated number of visible photons corresponding to each amount of delivered dose is calculated and shown in Fig. 6. For all the investigated field sizes, the ISD shows excellent linearity from very low dose to high dose values as described by linear regression analysis $\left(\mathrm{R}^{2}=1\right.$ and 0.9998$)$ and employing a linear fit.

Measurements show that the average deviations from the fitted curve are less than $0.53 \%$ for the $10 \times 10 \mathrm{~cm}^{2}$ field and less than $1.1 \%$ for the $1 \mathrm{x} 1 \mathrm{~cm}^{2}$ field. The maximum deviations were observed at the very low dose value (shown in insert) that can be due to the lack of exact dose

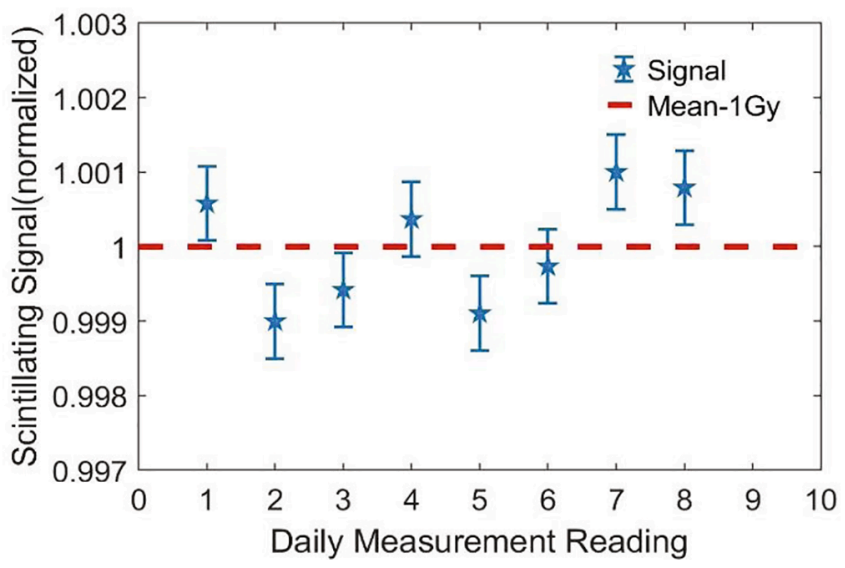

Fig. 5. Daily reproducibility of the ISD over eight consecutive days. Measurements normalized to an average of 1 . delivery by the LINAC machine at this small amount of dose value.

\section{Percentage depth dose distribution}

Fig. 7 represents the PDD profile measurements with the ISD for different beam field sizes. All curves exhibit the same global behavior with the maximum depth dose position approximately $15 \mathrm{~mm}$ as expected at the $6 \mathrm{MV}$ beam $[25,26,34,42]$. However, some discrepancies were observed after maximum depth dose, as entry doses are increasing with the field size due to the increase of the diffusion volume and scattering electrons coming from the LINAC head.

A comparison of PDD distribution between the ISD and microdiamond detector is shown in Fig. 8. For the investigated beam size of 10 $\mathrm{x} 10 \mathrm{~cm}^{2}$ and $0.5 \times 0.5 \mathrm{~cm}^{2}$, the average percentage difference as a residual is held at $1.30 \%$ and $2.94 \%$, respectively. Therefore, good agreement was observed in both cases. However, as the scintillator is not a water equivalent material, hence the scattered radiation increases with phantom depth, and a little discrepancy appeared at the build-down region. Moreover, the higher difference was observed in the small field, a reverse behavior than what we observed before [34], which can be attributed to the different sensitivities of each detector to incident and secondary photons in addition to other charged particles.

\section{Relative dose response with field size}

The photon flux recorded by the photon counter as a function of time is presented in Fig. 9(a) for field sizes ranging from $0.5 \times 0.5 \mathrm{~cm}^{2}$ to 10 $\times 10 \mathrm{~cm}^{2}$, respectively. All the curves show similar behavior with a rapid increase, followed by a beam stabilization step (a plateau) ending with a rapid fall-down while the beam switched off. As the photon counter rise time is in the ns range, the longer rise time observed is due to LINAC beam stabilization and characteristics of the scintillator $[43,44]$. The maximum signal collected by the counter increases with field size. The total number of photons during each beam field is equal to the total integral of the visible photon variation peaks observed in Fig. 9(a). Note that, in each case, the integrated optical signal (shown in Fig. 9b) is obtained by subtraction of the stem signal from the total recorded signal, which corresponds to the actual scintillating signal during irradiation. Finally, these photons were converted to respective dose values by using the calibration coefficient of the ISD. It observed that the response of the ISD is field-dependent, which indicates that a detector specific field correction is required to calculate the absolute dose at different fields.

\section{Field output factor comparison}

Fig. 10 shows the variations of field output factor as a function of effective field size for the ISD and microdiamond, where the curves have been normalized regarding the signal value obtained for a field size of 


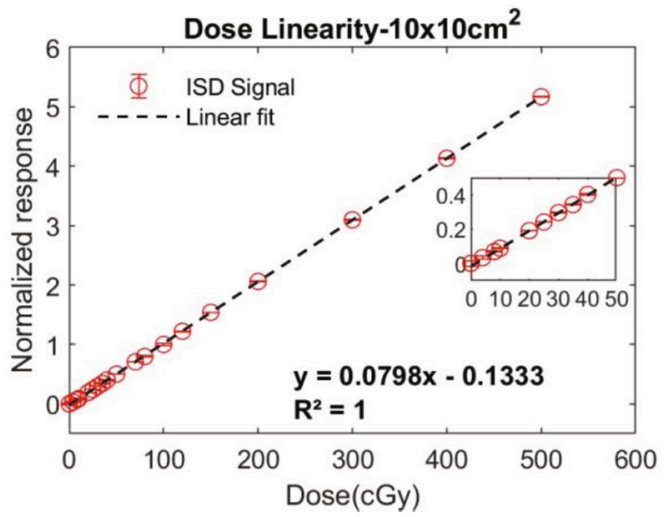

(a)

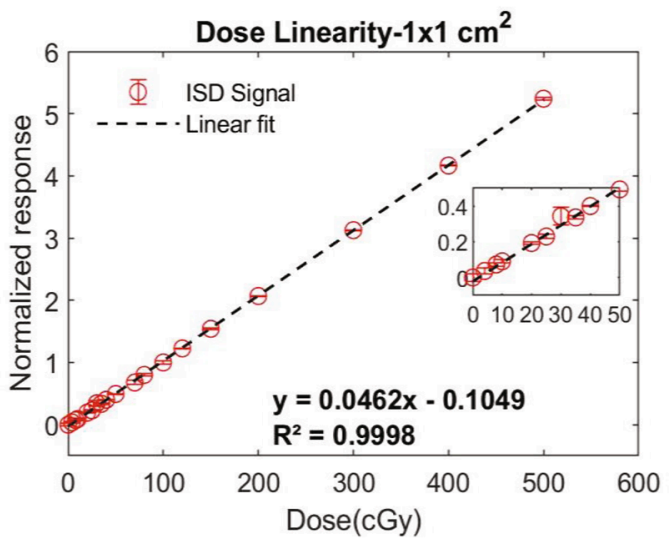

(b)

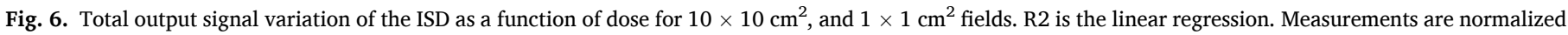
at the dose of $100 \mathrm{cGy}$.

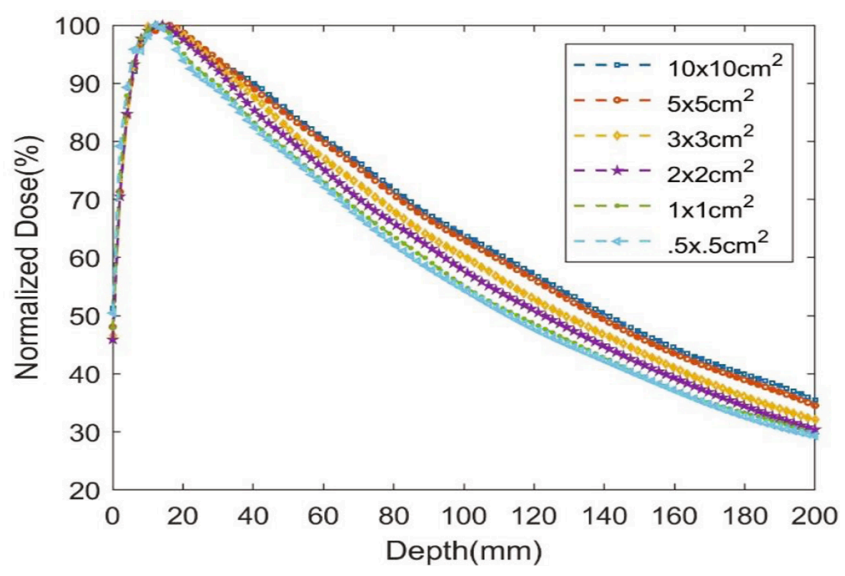

Fig. 7. PDD distribution by the ISD obtained for $10 \times 10 \mathrm{~cm}^{2}, 5 \times 5 \mathrm{~cm}^{2}, 3 \times 3$ $\mathrm{cm}^{2}, 2 \times 2 \mathrm{~cm}^{2}, 1 \times 1 \mathrm{~cm}^{2}$, and $0.5 \times 0.5 \mathrm{~cm}^{2}$ fields.

$10 \times 10 \mathrm{~cm}^{2}$. The field output factor decreases with the field size decreasing and achieves 0.55 and 0.48 for the ISD and microdiamond, respectively at the smallest irradiation field. The field output factor of the ISD is lower than the microdiamond until $1 \times 1 \mathrm{~cm}^{2}$ field size, whereas, for the lowest dimension field, this behavior is reversed. This can be attributed to the higher sensitivity of the ISD sensor to lower energy particles and the possible volume averaging issue of microdiamond at this field dimension. A little discrimination can partially be attributed to a slight misalignment between each detector with respect to the field center. Moreover, as the scintillator used in the ISD is not water equivalent and the result shown here does not consider any correction factor, thus discrimination in the field output comparison could be expected for different fields.

\section{Stem effect characterizations}

The stem effect has been measured as a function of fiber length following the description in section 2.3 , and the results of this study are presented in Fig. 11. This figure shows the relative contribution of this parasitic effect regarding the total signal measured (in \% of the stem to signal). We observed that this effect increases linearly with field size. Indeed, it is expected to be proportional to the irradiated fiber volume and thus to the fiber length within the beam. Besides, the results also highlight that for the field sizes $3 \times 3 \mathrm{~cm}^{2}$ to $0.5 \times 0.5 \mathrm{~cm}^{2}$, the contribution of this effect to the total optical signal magnitude becomes less than $1 \%$.

\section{Uncertainty budget}

Table 1 summarizes the uncertainty budget for the ISD and microdiamond measurements for the experimental results of PDD, beam profile, and field output factors. The uncertainties were evaluated by statistical analysis in the measurement (standard deviation in the readings- type A) and detectors' positioning uncertainty (type B).

\section{Discussion}

In this study, the optical fiber integrated ISD system shows that measurements are reproducible within (0.02-0.08) \% successive and $0.1 \%$ daily measurements as demonstrated during experiments. These

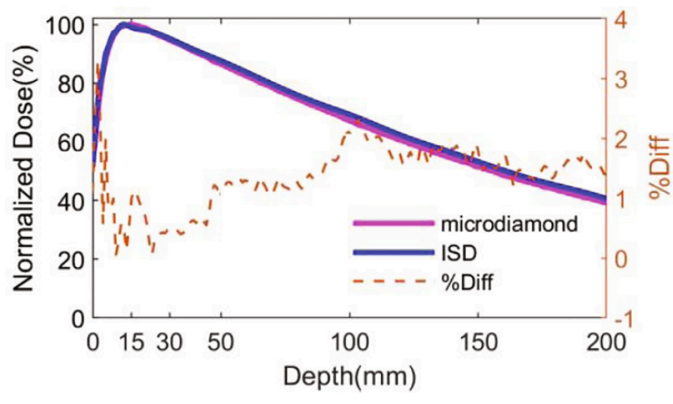

(a)

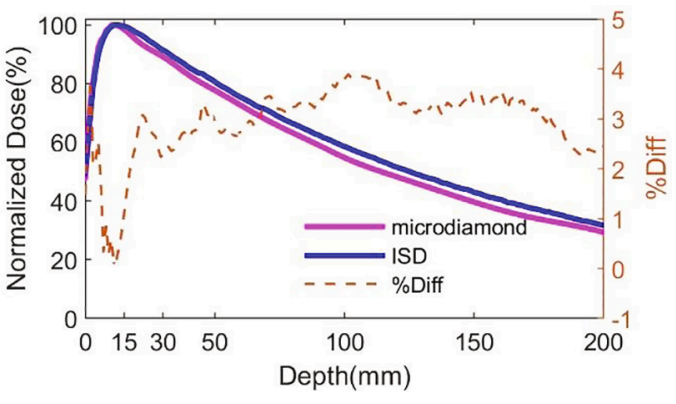

(b)

Fig. 8. PDD comparison between the ISD and microdiamond for (a) $10 \times 10 \mathrm{~cm}^{2}$ and (b) $0.5 \times 0.5 \mathrm{~cm}^{2}$ field. Residuals are given in percentage and shown in right side (brown color). 


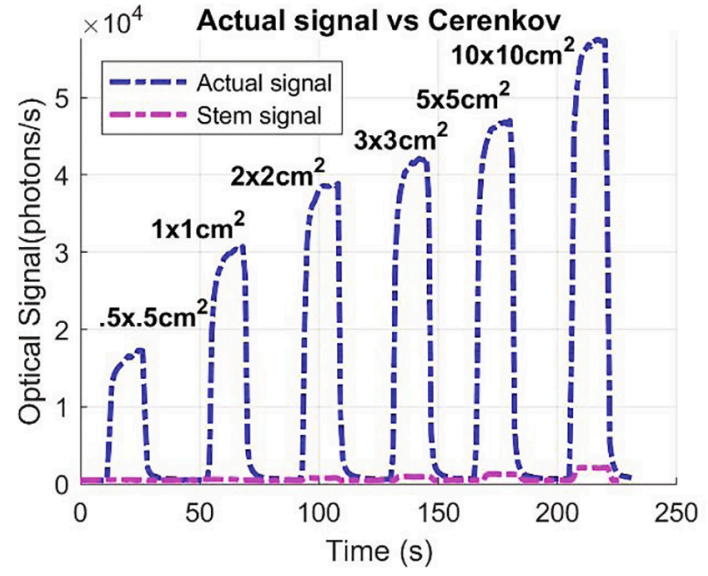

(a)

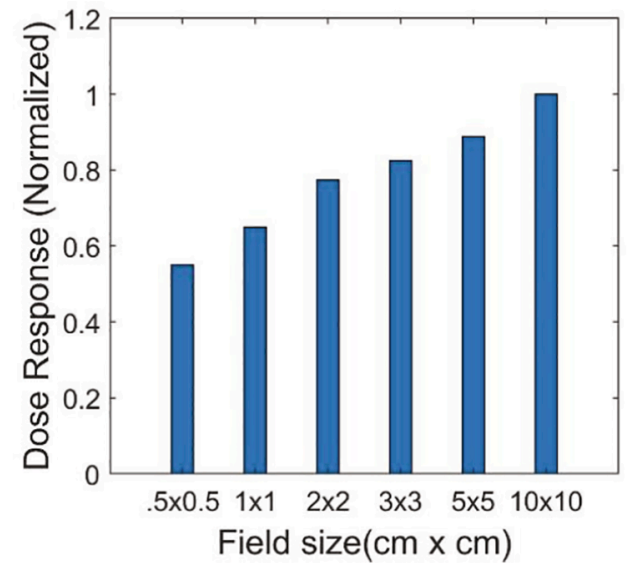

(b)

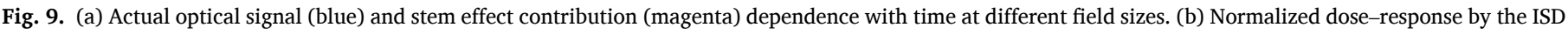

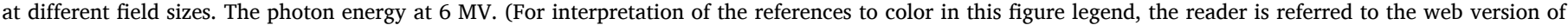
this article.)

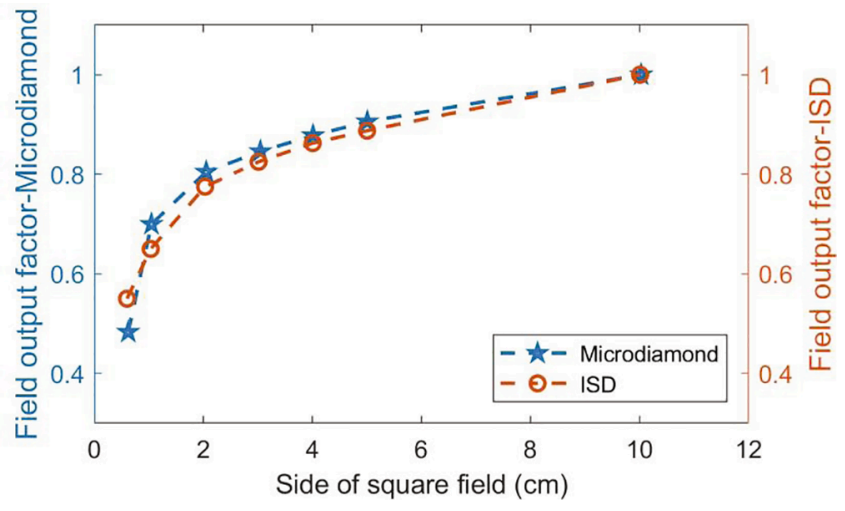

Fig. 10. Field output factor for microdiamond and the ISD at different beam fields $\left(10 \times 10 \mathrm{~cm}^{2}\right.$ to $\left.0.5 \times 0.5 \mathrm{~cm}^{2}\right)$. The side of the square field is presented based on effective (measured) field size.

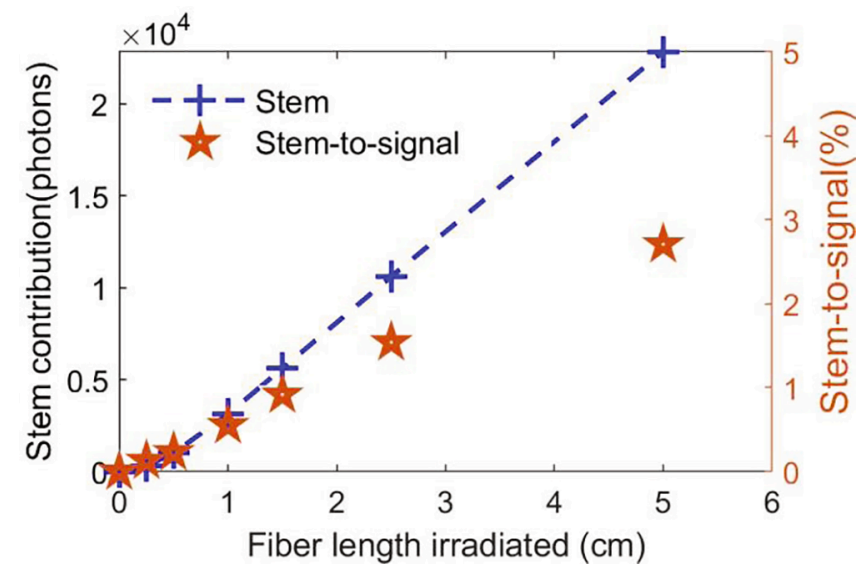

Fig. 11. Stem contribution of the ISD detector with respect to the irradiated fiber lengths. The left scale represents the total stem effect recorded (blue curve); the brown dots represent the percentage of stem-to-signal ratio recorded as can be seen from the right scale. (For interpretation of the references to color in this figure legend, the reader is referred to the web version of this article.)
Table 1

Measurement uncertainties estimation for PDD, beam profiles and output factors.

\begin{tabular}{|c|c|c|}
\hline \multicolumn{3}{|c|}{ PDD and beam profiles } \\
\hline Source & $\begin{array}{l}\text { Uncertainty } \\
\text { Contribution (ISD) }\end{array}$ & $\begin{array}{l}\text { Uncertainty Contribution } \\
\text { (Microdiamond) }\end{array}$ \\
\hline $\begin{array}{l}\text { Measurement } \\
\text { repeatability (A) }\end{array}$ & $0.15 \%$ & $0.10 \%$ \\
\hline Positioning (B) & $0.20 \%$ & $0.25 \%$ \\
\hline \multicolumn{3}{|l|}{ Field output factor } \\
\hline Source & & \\
\hline $\begin{array}{l}\text { Measurement } \\
\text { repeatability (A) }\end{array}$ & $0.10 \%$ & $0.15 \%$ \\
\hline Positioning (B) & $0.20 \%$ & $0.20 \%$ \\
\hline
\end{tabular}

results explore the detector's excellent stability under the small field irradiation, which is a very significant outcome when compared to the value achieved in some recent studies [28,29,31]. As seen from the linear regression and linear fit analysis, the detector provides entire proportional behavior to a very low (4 cGy) to high dose (500 cGy) within average deviations less than $0.53 \%$ and $1.10 \%$, respectively from the linear fit for different fields considered in this work. This result is of great importance for further calibration steps of the device required to achieve a direct dose reading.

At the range of $6 \mathrm{MV}$ photons, the absorption coefficient $\mu$ is about $2.10^{-2} \mathrm{~cm}^{-1}$ in water [45]. So, at water depths ranging from 0 to $20 \mathrm{~cm}$, photon flux from the LINAC source is high, therefore, generates more charged particles and low energy X-rays. Thus, increasing the irradiated volume (while increasing the field size), the number of generated particles close to the detector is increasing. That is why PDD profiles for the ISD in the build-down region decrease more slowly when the field size increases. On the other hand, the over-response of the ISD in the builddown region could be attributed to its sensitivity to low energy photon generates from secondary emission or scattered radiation. A reverse behavior was observed while comparing it to our previous result [34]. It signifies that the sensitivity of this new scintillator is higher in small fields.

Due to the small convolution effect between field and detector shapes, a little discrepancy of FWHM $(\sim 0.3 \mathrm{~mm})$ regarding the selected field size $\left(0.5 \times 0.5 \mathrm{~cm}^{2}\right)$ was observed in the air by the ISD. Besides, the wider dimension measured in water (FWHM $\sim 6 \mathrm{~mm}$ ) by the ISD can be attributed to the detection of secondary X-ray photons, and charged particles created beyond the field edges. Contrarily, using the microdiamond detector, the FWHM of $7 \mathrm{~mm}$ inside water is reasonably due to 
the bigger convolution effect. In addition, $20 \%$ of the maximum signal is achieved for the ISD when the detector is positioned at $4 \mathrm{~mm}$ from the field center, while the same value is reached at $5.5 \mathrm{~mm}$ for the microdiamond. This position difference of $1.5 \mathrm{~mm}$ corresponds to approximately half the size of the microdiamond dosimeter $\left(0.004 \mathrm{~mm}^{3} ; 4 \mathrm{~mm}^{2}\right.$ active measurement area). It highlights the drastic contribution of the convolution effect when characterizing small fields using dosimeters of significant dimensions.

The spatial resolution of the ISD was shown to be $100 \mu \mathrm{m}$ that demonstrates through dose measurement discrimination between two neighboring points, which is slightly better than a recently developed $2 \mathrm{D}$ monolithic silicon array detector [46]. The scintillating signal amplitude difference between two successive positions in the fall-down region of the field lateral profile is about 500 photons/s, while the sensitivity of the photon counter is 20 photons/s. Thus, the spatial resolution of the ISD is apparently better than $100 \mu \mathrm{m}$ and can certainly be further improved by decreasing the fiber core diameter. It will separate penumbra from the convolution effects in small fields, owing to the high signal-to-noise ratio of the ISD.

The stem contribution decreases with the fiber length irradiated and reaches less than $1 \%$ of the signal magnitude for field sizes below $3 \times 3$ $\mathrm{cm}^{2}$ that is still significant for scintillating dosimetry with such a small detector. Even though the contribution of the stem signal to the actual optical signal is very low, this parasitic effect was systematically quantified and suppressed from the total signal measured.

Field output factor was reported as a function of effective field size since both detectors (ISD and microdiamond) do not measure the same FWHM in both inline and crossline direction at the very small field. It simplifies the measured field width into one representative value. The ISD shows consistency with microdiamond while comparing field output factor measurement. Some discrepancies were expected here, as both detectors did not measure the same effective field for the given nominal field size. Note that no correction factor was considered for either the ISD or microdiamond, as it was a relative dose measurement. Knowing that for the ISD, it requires Monte Carlo (MC) simulation that was not considered in this study. Therefore, field output factor variation with respect to microdiamond stays within $4.5 \%$ for $0.5 \times 0.5 \mathrm{~cm}^{2}$ field and within $1.5 \%$ for $1 \times 1 \mathrm{~cm}^{2}$ to larger fields. However, considering the consistency in field output measurement, ISD may provide appropriate dose-response at different small fields.

For the PDD and beam profiling, the total measurement uncertainty estimation is well below $0.3 \%$, and for the output factor, the uncertainty evaluated is within $0.25 \%$, which is still in the range of typical dosimetric requirements. However, this measurement uncertainty can still be reduced by increasing the number of repeated measurements and simultaneous acquisitions.

\section{Conclusion}

In this study, we have shown the dosimetric characteristics of a new fiber-integrated X-ray inorganic detector with a demonstrated spatial resolution of $100 \mu \mathrm{m}$. The detector provides an entire linear response with the dose in 0 to $500 \mathrm{cGy}$ range, independently of the field size selected between $10 \times 10 \mathrm{~cm}^{2}$ and $0.5 \times 0.5 \mathrm{~cm}^{2}$. Perfect repeatability (less than $0.07 \%$ difference from average values) with good day-to-day reproducibility (maximum $0.1 \%$ difference from average values) demonstrates its feasibility in the radiotherapy application under regular to small field irradiation. The issue of stem effect that usually affects optical fiber scintillating measurements has been addressed and demonstrated that the developed system is essentially free from this effect at the small fields e.g., below $3 \times 3 \mathrm{~cm}^{2}$ field. So, dose measurement under small field irradiation can be more accurate than using conventional scintillating dosimeters.

The ISD system showed better accuracy than microdiamond when comparing the small field beam profile measurement, thanks to the lower convolution effect induced due to the miniature size detector having the smallest sensitive volume. A cross-section lateral profile of the smallest field $\left(0.5 \times 0.5 \mathrm{~cm}^{2}\right)$ was obtained with a high spatial resolution to observe the accuracy in beam profile measurement.

PDD distribution of the ISD at various small fields suggested that the detector might be eligible to measure the accurate relative dose at different depths inside the water. However, further investigations are needed to address the discrepancies in the build-down region of the smallest field. Moreover, measurement reveals that the ISD provides a high signal-to-noise ratio, good stability of the signal with accumulated dose, and minor contamination with Cerenkov light that leads to a more widespread application in the online dose verification system. In agreement with the obtained results, it can be possible to shrink down the detector volume while keeping a significant luminescence signal at the output with good sensitivity. Therefore, a very high spatial resolution sensor with a few micro-meter scintillating heads could be achieved that cannot be achieved by the conventional detector so far.

The estimated ISD measurement uncertainty was found to be in an acceptable range for dosimetry application. Finally, considering the performance of the ISD in different small fields, it is expected that the ISD can be an efficient alternative in the current radiotherapy detection technique.

\section{Acknowledgements}

This project has received funding from the European Union's Horizon 2020 Research and Innovation Program under the Marie Skłodowska-Curie grant agreement No.713750. Also, it has been carried out with the financial support of the Regional Council of Provence- AlpesCôte d'Azur and with the financial support of the A*MIDEX ( ${ }^{\circ}$ ANR- 11IDEX-0001-02), funded by the Investissements d'Avenir project funded by the French Government, managed by the French National Research Agency (ANR). This work was also supported by the french government under the Programme Investissements d'Avenir, Initiative d'Excellence d'Aix-Marseille Université - A*Midex AMX-18-UNT-018.

\section{References}

[1] Vordermark D. Ten years of progress in radiation oncology. BMC Cancer 2011;11 (1). https://doi.org/10.1186/1471-2407-11-503.

[2] Kron T, Lehmann J, Greer PB. Dosimetry of ionising radiation in modern radiation oncology. Phys Med Biol 2016;61(14):R167-205.

[3] Rosenfeld AB, Biasi G, Petasecca M, Lerch ML, Villani G, Feygelman V. Semiconductor dosimetry in modern external-beam radiation therapy. Phys Med Biol 2020, 65:16TR01. https://doi.org/10.1088/1361-6560/aba163.

[4] Gagnon J-C, Thériault D, Guillot M, Archambault L, Beddar S, Gingras L, Beaulieu L. Dosimetric performance and array assessment of plastic scintillation detectors for stereotactic radiosurgery quality assurance: Dosimetric performance, array assessment of PSD for SRS. Med Phys 2012;39(1):429-36.

[5] 91 IRN.Prescribing, recording, and reporting of stereotactic treatments with small photon beams. J ICRU 2014, 14:1-160. https://dx.doi.org/10.1093/jicru/ndx017.

[6] Vieillevigne L, Arnaud FX. Dosimetric performance of the new PTW 31022 PinPoint 3D ionization chamber in high energy photon beams. Biomed Phys Eng Express 2018, 4:047002. https://dx.doi.org/10.1088/2057-1976/AABEEF.

[7] Kržanovic N, Živanovic M, Ciraj-Bjelac O, Lazarevic Đ, Ceklic S, Stankovic S. Performance testing of selected types of electronic personal dosimeters in X-and gamma radiation fields. Health Phys 2017;113:252-61. https://doi.org/10.1097/ hp.0000000000000704.

[8] Petoukhova A, Rüssel I, Nijst-Brouwers J, van Wingerden Ko, van Egmond J, Jacobs D, Marinelli A, van der Sijp J, Koper P, Struikmans H. In vivo dosimetry with MOSFETs and GAFCHROMIC films during electron IORT for Accelerated Partial Breast Irradiation. Phys Med 2017;44:26-33.

[9] Le Roy M, de Carlan L, Delaunay F, Donois M, Fournier P, Ostrowsky A, Vouillaume A, Bordy JM. Assessment of small volume ionization chambers as reference dosimeters in high-energy photon beams. Phys Med Biol 2011;56(17): $5637-50$.

[10] Reggiori G, Stravato A, Mancosu P, Lobefalo F, Paganini L, Zucconi F, Palumbo V, Gaudino A, Scorsetti M, Tomatis S. Small field characterization of a Nanochamber prototype under flattening filter free photon beams. Phys Med 2018;49:139-46.

[11] Casar B, Gershkevitsh E, Mendez I, Jurković S, Saiful Huq M. Output correction factors for small static fields in megavoltage photon beams for seven ionization chambers in two orientations - Perpendicular and parallel. Med Phys 2020;47(1): 242-59.

[12] Palmans H, Andreo P, Huq MS, Seuntjens J, Christaki K. In: Dosimetry of small static fields used in external beam radiotherapy: An IAEA-AAPM international code 
of practice for reference and relative dose determination. Vienna: International Atomic Energy Agency; 2017. https://doi.org/10.1002/mp.13208.

[13] Francescon P, Cora S, Cavedon C, Scalchi P, Reccanello S, Colombo F. Use of a new type of radiochromic film, a new parallel-plate micro-chamber, MOSFETs, and TLD 800 microcubes in the dosimetry of small beams. Med Phys 1998;25(4):503-11.

[14] Eklund K, Ahnesjö A. Modeling silicon diode energy response factors for use in therapeutic photon beams. Phys Med Biol 2009;54(20):6135-50.

[15] Yin Z, Hugtenburg RP, Beddoe AH. Response corrections for solid-state detectors in megavoltage photon dosimetry. Phys Med Biol 2004;49(16):3691-702.

[16] Griessbach I, Lapp M, Bohsung J, Gademann G, Harder D. Dosimetric characteristics of a new unshielded silicon diode and its application in clinical photon and electron beams: New unshielded silicon diode. Med Phys 2005;32(12): $3750-4$.

[17] Parwaie W, Refahi S, Ardekani MahdiehAfkhami, Farhood B. Different dosimeters/ detectors used in small-field dosimetry: Pros and cons. J Med Signals Sens 2018;8 (3):195. https://doi.org/10.4103/jmss.JMSS 3 18.

[18] Palmer AL, Dimitriadis A, Nisbet A, Clark CH. Evaluation of Gafchromic EBT-XD film, with comparison to EBT3 film, and application in high dose radiotherapy verification. Phys Med Biol 2015;60(22):8741-52.

[19] Yarahmadi M, Nedaie H, Allahverdi M, Asnaashari K, Sauer O. Small photon field dosimetry using EBT2 Gafchromic film and Monte Carlo simulation. Int J Radiat Res 2013;11:215. https://ijrr.com/article-1-1101-en.html.

[20] Pai S, Das IJ, Dempsey JF, Lam KL, LoSasso TJ, Olch AJ, Palta JR, Reinstein LE, Ritt D, Wilcox EE. TG-69: Radiographic film for megavoltage beam dosimetry: TG69: Radiographic film. Med Phys 2007;34(6Part1):2228-58.

[21] Brace OJ, Alhujaili SF, Paino JR, Butler DJ, Wilkinson D, Oborn BM, Rosenfeld AB, Lerch MLF, Petasecca M, Davis JA. Evaluation of the PTW microDiamond in edgeon orientation for dosimetry in small fields. J Appl Clin Med Phys 2020;21(8): 278-88.

[22] Das IJ, Morales J, Francescon P. Small field dosimetry: What have we learnt? In: AIP Conference Proceedings: AIP Publishing LLC; 2016. https://dx.doi.org/ $10.1063 / 1.4954111$.

[23] De Coste V, Francescon P, Marinelli M, Masi L, Paganini L, Pimpinella M, Prestopino G, Russo S, Stravato A, Verona C. Is the PTW 60019 microDiamond a suitable candidate for small field reference dosimetry? Phys Med Biol 2017;62: 7036. https://doi.org/10.1088/1361-6560/aa7e59.

[24] Therriault-Proulx F, Pino R, Yang JN, Beddar AS. Quality assurance for Gamma Knife Perfexion using the Exradin W1 plastic scintillation detector and Lucy phantom. Phys Med Biol 2019;64:225007. https://doi.org/10.1088/1361-6560/ ab4ac3.

[25] Galavis PE, Hu L, Holmes S, Das IJ. Characterization of the plastic scintillation detector Exradin W2 for small field dosimetry. Med Phys 2019;46(5):2468-76.

[26] Beaulieu L, Beddar S. Review of plastic and liquid scintillation dosimetry for photon, electron, and proton therapy. Phys Med Biol 2016;61(20):R305-43.

[27] Archambault L, Beddar AS, Gingras L, Roy R, Beaulieu L. Measurement accuracy and Cerenkov removal for high performance, high spatial resolution scintillation dosimetry: Measurement accuracy and Cerenkov removal for scintillation dosimetry. Med Phys 2006;33(1):128-35.

[28] Martínez N, Rucci A, Marcazzó J, Molina P, Santiago M, Cravero W. Characterization of YVO 4:Eu 3+ scintillator as detector for Fiber Optic Dosimetry. Radiat Meas 2017;106:650-6.
[29] Alharbi M, Gillespie S, Woulfe P, Mccavana P, O’Keeffe S, Foley M. Dosimetric characterization of an inorganic optical fiber sensor for external beam radiation therapy. IEEE Sens J 2019;19(6):2140-7.

[30] Debnath SBC, Ferre M, Tonneau D, Fauquet C, Tallet A, Goncalves A, et al. High resolution small-scale inorganic scintillator detector: HDR brachytherapy application. Med Phys 2021. https://doi.org/10.1002/mp.14727.

[31] Carrasco P, Jornet N, Jordi O, Lizondo M, Latorre-Musoll A, Eudaldo T, Ruiz A, Ribas M. Characterization of the Exradin W1 scintillator for use in radiotherapy: Characterization of Exradin W1 for radiotherapy. Med Phys 2015;42(1):297-304.

[32] Kertzscher G, Beddar S. Inorganic scintillation detectors based on Eu-activated phosphors for 192 Ir brachytherapy. Phys Med Biol 2017;62(12):5046-75.

[33] Beddar AS. Plastic scintillation dosimetry and its application to radiotherapy. Radiat Meas 2006;41:S124-33.

[34] Debnath SBC, Fauquet C, Tallet A, Goncalves A, Lavandier S, Jandard F, Tonneau D, Darreon J. High spatial resolution inorganic scintillator detector for high-energy X-ray beam at small field irradiation. Med Phys 2020;47(3):1364-71.

[35] Safai S, Lin S, Pedroni E. Development of an inorganic scintillating mixture for proton beam verification dosimetry. Phys Med Biol 2004;49(19):4637-55.

[36] Kaur J, Dubey V, Suryanarayana NS. Comparative study of ML and PL spectra of different impurity-doped (Zn, Cd)S mixed phosphors. Res Chem Intermed 2013;39 (9):4337-49.

[37] Liu PZY, Suchowerska N, Lambert J, Abolfathi P, McKenzie DR. Plastic scintillation dosimetry: Comparison of three solutions for the Cerenkov challenge. Phys Med Biol 2011;56(18):5805-21.

[38] Beddar AS, Mackie TR, Attix FH. Water-equivalent plastic scintillation detectors for high-energy beam dosimetry: I. Physical characteristics and theoretical considerations. Phys Med Biol 1992;37(10):1883-900.

[39] JCGM J, 2008. Evaluation of measurement data-Guide to the expression of uncertainty in measurement. Int. Organ. Stand. Geneva ISBN 2008, 50:134.

[40] Musolino SV. Absorbed dose determination in external beam radiotherapy: An international code of practice for dosimetry based on standards of absorbed dose to water; technical reports series no. 398. Health Phys 2001;81(5):592-3.

[41] Cranmer-Sargison G, Charles PH, Trapp JV, Thwaites DI. A methodological approach to reporting corrected small field relative outputs. Radiother Oncol 2013; 109(3):350-5.

[42] Al Mashud MA, Tariquzzaman M, Alam MJ, Zakaria G. Photon beam commissioning of an Elekta Synergy linear accelerator. Pol J Med Phys Eng 2017, 23:115-19. https://dx.doi.org/10.1515/pjmpe-2017-0019.

[43] Alharbi M, Martyn M, O'Keeffe S, Therriault-Proulx F, Beaulieu L, Foley M. Benchmarking a novel inorganic scintillation detector for applications in radiation therapy. Phys Med 2019;68:124-31.

[44] Ramírez M, Martínez N, Marcazzó J, Molina P, Feld D, Santiago M. Performance of ZnSe(Te) as fiberoptic dosimetry detector. Appl Radiat Isot 2016;116:1-7.

[45] Commerce USDo. National Institute of Standards and Technology, NIST. Available at: https://physics.nist.gov/PhysRefData/Xcom/html/xcom1.html [accessed 10 July 2020].

[46] Biasi G, Petasecca M, Guatelli S, Hardcastle N, Carolan M, Perevertaylo V, Kron T, Rosenfeld AB. A novel high-resolution 2D silicon array detector for small field dosimetry with FFF photon beams. Phys Med 2018;45:117-26. 